\title{
Habitar la naturaleza en armonía con el universo. Metafísica, geometría cósmica y orden social en la tradición arquitectónica china
}

\section{Inhabiting Nature in Harmony with the Universe. Metaphysics, Cosmic Geometry and Social Order in the Chinese Architectural Tradition}

\author{
José Manuel Almodóvar Melendo ${ }^{1}$ \\ Yingying $\mathrm{Xu}^{2}$ \\ Universidad de Sevilla (España)
}

Recibido: 10-01-16

Aprobado: 18-02-16

\section{Resumen}

En este artículo se establece un paralelismo entre la arquitectura tradicional china y concepciones cosmológicas y sociales, con objeto de analizar su proceso de formalización. En este sentido, se discute el modo en que los patrones arquitectónicos se han estandarizado a partir de módulos o elementos relacionados en diferentes niveles de composición y articulados en torno al vacío, que simboliza la naturaleza, para alcanzar el equilibrio con el Cosmos. Las conclusiones evidencian que se han establecido unas relaciones llenas de significado entre el hombre y la naturaleza, que responden simultáneamente a una síntesis de ideologías y filosofías jerarquizadas que provienen del confucianismo y su continuo desarrollo.

Palabras-clave: Arquitectura tradicional china, Confucianismo, Patrones arquitectónicos, Armonía con la naturaleza

\footnotetext{
${ }^{1}$ (jmalmodovar@us.es). Profesor Titular en la Universidad de Sevilla. Doctor Arquitecto por la Universidad de Sevilla (2000) y Máster en Energías Renovables (2000) por la Universidad Internacional de Andalucía. Ha obtenido el Título del Instituto de Idiomas de la Universidad de Sevilla de Chino (2012), Japonés (2008) y Portugués (2000). Actualmente es profesor en el Grado en Estudios de Asia Oriental y ETS de Arquitectura, Universidad de Sevilla. Ha sido investigador invitado en Tsinghua University (Pekín), Kobe Design University (Japón), Columbia University (New York), Cal Poly Pomona (Los Angeles) y University of Berkeley California. Así mismo, ha dirigido o participado en proyectos de investigación financiados por la Unión Europea y en colaboración con universidades Asia, EEUU e Iberoamérica.

${ }^{2}$ (xying@us.es). Profesora Sustituta Interina de chino en el Grado en Estudios de Asia Oriental. Licenciada y Doctoranda en Arquitectura y Máster en Traducción e Interculturalidad por la Universidad de Sevilla. Ha obtenido el Certificado de Profesor del idioma chino otorgado por la Oficina de Asuntos Extranjeros Chinos del Consejo de Estado de China.
} 


\section{Abstract}

In this paper, a parallelism between traditional Chinese architecture and cosmological and social conceptions is established to analyze its process of formalization. In this regard, it is discussed the way in which architectural patterns have been standardized from modules or elements related in various levels of composition and articulated around the vacuum, that symbolizes nature, to achieve equilibrium with the Cosmos. The conclusions show that meaningful relationships between man and nature have been established that simultaneously reproduce a hierarchical synthesis of ideologies and philosophies that come from Confucianism and its continuous development.

Key-words: Traditional Chinese architecture, Confucianism, Architectural patterns, Harmony with nature.

\section{Introducción}

La arquitectura no ha sido considerada tradicionalmente en China como un tipo de arte, aunque evidentemente se trata de una de sus manifestaciones culturales más significativas. De hecho, el concepto de forma o xíng 形 ha estado vinculado a la ejecución de modelos establecidos, mientras que el nivel de conocimiento que está más allá de la formalización o ejecución, se ha situado en el campo de la filosofía o las artes. En este sentido cabe mencionar, que para hacer referencia a los edificios se utiliza un término ligado a la ejecución, en concreto tǔmù 土木, que significa literalmente tierra-madera. Así mismo, el conocimiento sobre arquitectura ha estado ligado a manuales prácticos, tales como el Yíngzàofăshi 營造法式 o Normas de Construcción del Estado. Estos conceptos pueden verse reflejados en torno al 1100 a.C. en el Yijing 易经 o libro de los cambios, en el que se comenta que:
形而上者谓之道, 形而下者谓之器。
Xing 'érshàng zhě wèi zhī dào, xíng 'érxià zhě wèi zhī qì.
Lo que está por encima de la forma se llama Tao, lo que está debajo de la forma se llama objeto.

Conviene aclarar, que dào 道 es el término utilizado en la tradición china para hacer referencia al orden natural. Podemos deducir, por tanto, que la formalización ha estado vinculada a la construcción o práctica, aunque paralelamente se ha establecido una correspondencia entre arquitectura y conceptos cosmológicos. Por tanto, se ha articulado una forma de pensamiento correlativo mediante la cual, la arquitectura se ha considerado como un microcosmos que debe de reflejar el macrocosmos. Este paralelismo ha estado 
ligado a su vez a la concepción de que existe una correspondencia entre el orden celestial y el de la tierra. En este sentido, se ha entendido tradicionalmente que "el cielo es redondo y la tierra cuadrada", “天圆地方 tiān yuán dì fāng” (Henderson 2011: 61).

Podemos deducir, por tanto, que las bases conceptuales que condicionan la formalización de los patrones arquitectónicos chinos ya estaban establecidas hace más de tres mil años. Al haberse configurado en una etapa tan temprana presentan unas relaciones indisolubles con la cultura, el pensamiento y la sociedad. En este sentido, conviene aclarar que aunque China sea un país con una diversidad regional y étnica considerable, presenta también fuertes tendencias de coherencia cultural que se reflejan en su arquitectura. Como consecuencia, y aunque ha existido una lógica evolución en la arquitectura china, su desarrollo ha sido lento, manteniéndose una continuidad en aspectos esenciales. En este sentido, Liáng Sïchéng 梁思成 comenta que:

It is an indigenous growth that was conceived and born in the remote prehistoric past, reached its "adolescence" in the Han dynasty, matured into full glory and vigor in the Tang Dynasty, mellowed with grace and elegance in the Song dynasty, then started to show sign of old age, feebleness, and rigidity.

Es un crecimiento autóctono que fue concebido y nació en el pasado prehistórico remoto, alcanzó su "adolescencia" en la dinastía Han, maduró lleno de gloria y vigor en la dinastía Tang, fue suavizado con gracia y elegancia en la dinastía Song, y posteriormente comenzó a mostrar signos de envejecimiento, debilidad y rigidez (Sicheng 2010: 65).

Debido a la existencia de una concepción ancestral, según la cual, la arquitectura debe estar en correspondencia con el orden natural, el sistema de composición y organización espacial chino ha estado vinculado al desarrollo de geometrías cósmicas, basadas en relaciones numéricas, que eran utilizadas para explicar la naturaleza del cosmos. Como consecuencia, se estableció una relación armónica entre el hombre y la naturaleza, cuestión que ha sido perseguida constantemente en la historia de la filosofía.

En este sentido, en China se ha considerado que el hombre es sólo una parte de la naturaleza, que alcanza su sentido o razón de ser en su integración con ella. Por tanto, la naturaleza se ha situado jerárquicamente por encima del hombre. Por el contrario en Occidente, en términos generales, se ha considerado que el hombre es el ser superior de la naturaleza, y todo lo que le rodea está a su servicio.

De forma paralela a esta vinculación con la naturaleza, la arquitectura ha sido un reflejo del orden social, que representa la armonía entre los hombres. En este sentido, la sociedad china se ha articulado en torno a unos códigos basados en conceptos confucianos, que establecen unas rígidas y jerarquizadas 
relaciones dentro de la sociedad y especialmente en el ámbito familiar. Queremos señalar en este sentido, que la familia tradicional china constituía en sí misma una micro-sociedad rígidamente jerarquizada, cuyo orden o estructura era reproducido espacialmente en la vivienda.

En definitiva, nos proponemos explorar en este artículo, el modo en que la arquitectura tradicional china se ha articulado, a partir de la modulación y estandarización de patrones arquitectónicos, en una búsqueda ancestral por reproducir el orden cósmico y social para estar en armonía con la naturaleza.

\section{Orden cósmico, geomancia y ecología mística}

\subsection{La dualidad yīnyáng 阴阳 y la constitución del cosmos. El bāguà 八卦 como representación del espacio-tiempo}

En el sustrato más básico de la cultura China subyace la creencia de que el hombre debe ocupar la naturaleza en armonía con el universo, constituido por dualidades o elementos contrapuestos y complementarios. Estas ideas ya aparecen recogidas hace más de tres mil años en el Yijīng 易经, el libro de los cambios, también conocido como libro de las adivinaciones. De hecho, el pictograma 易 representa un animal bajo el sol, lo que sugiere la realización ancestral de sacrificios de animales con fines adivinatorios en fechas con simbología solar. Tanto el Taoísmo como el Confucianismo fueron influenciados en cierto modo por este libro, que encarna el espíritu de la cultura china. En efecto, una versión del Yijing anotada por Confucio es la que ha llegado hasta nuestros días, y alguno de los términos utilizados por Lăož fueron inspirados por él. En este clásico de la literatura china se comenta que:

易有太极, 是生两仪, 两仪生四象, 四象生八卦。

Yî yǒu tàijí, shì shēng liăng yí, liăng yí shēng sì xiàng, sì xiàng shēng bāguà.

Existe en los cambios el primer gran principio, esto genera las dos primeras fuerzas; las dos primeras fuerzas generan las cuatro imágenes, las cuatro imágenes generan los ocho trigramas.

Se sugiere por tanto, que el mundo está compuesto por dualidades. Las dos primeras fuerzas mencionadas en esta cita constituirían la primera dualidad, el yīn 阴 y el yáng 阳, que hacen referencia respectivamente a la puesta $\mathrm{y}$ 
salida del sol, oeste y este. A partir de esta polaridad derivada de la observación del movimiento del sol, se han desarrollado composiciones geométricas que representan ideas cosmológicas.

Los conceptos del yīn y el yáng, además del oeste y este, tienen un gran número de acepciones y aplicaciones: femenino-masculino, noche-día, parimpar, sólido-vacío, etc. Conviene aclarar que representan una dualidad que expresa a su vez una unidad implícita. Serían como las dos caras de una misma moneda, ya que están interrelacionados y a la vez son interdependientes.

El yīn se representa con una línea discontinua o débil, mientras que el yáng se identifica con una línea continua o fuerte. Combinando dos dualidades yīn-yáng se obtienen las $2^{2}=4$ imágenes citadas en el Yijjīng. De este modo se extiende la relación bilateral oeste-este a un modelo bidimensional de cuatro direcciones, en las que aparecen representadas la noción de norte y sur. Estas cuatro direcciones están asociadas a las cuatro estaciones: primavera-este, verano-sur, otoño-oeste e invierno-norte. Si a su vez se combinan con otra dualidad yīn-yáng, se obtienen $2^{3}=8$ trigramas, considerados los ocho símbolos primordiales que representan las energías constitutivas del cosmos (figura 1).

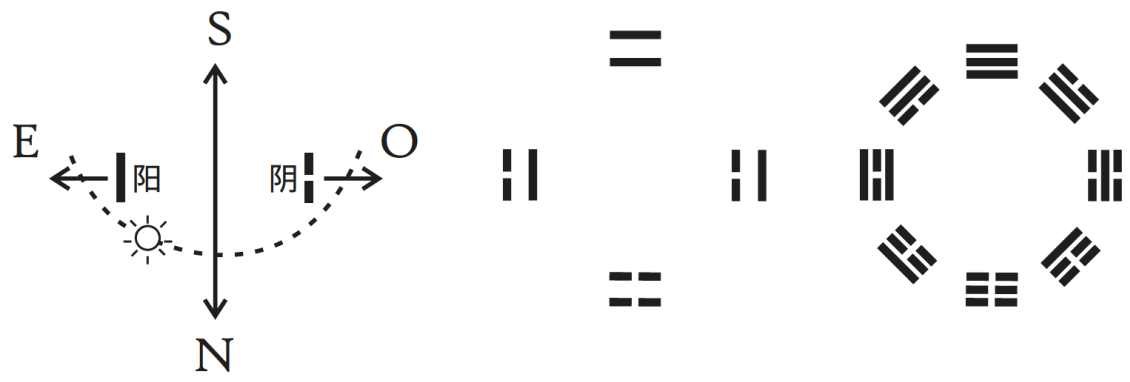

Figura 1. Configuración de las ocho direcciones a partir de la dualidad yīn-yáng 阴阳.

Como resultado de estas concepciones cosmológicas se genera un esquema conocido como bāguà 八卦. Significa literalmente ocho direcciones, ya que se considera que las orientaciones condicionan la forma en que fluyen las energías del universo, y que tienen por tanto una influencia decisiva para alcanzar la armonía con la naturaleza. Como hemos mencionado anteriormente, el yìn y el yáng están ligados a la trayectoria solar y por tanto al espacio (orientado respecto a la posición del sol) y al tiempo, marcado por su movimiento, que rige los ciclos día-noche y las cuatro estaciones. Como consecuencia, para buscar un equilibro entre el yīn-yáng u oeste-este, la arquitectura china ha ocupado tradicionalmente el espacio buscando una simetría con respecto al eje norte-sur, que le permite responder armónicamente al curso del tiempo. 


\subsection{La relación del hombre con la naturaleza. El concepto de centro}

El esquema cósmico anteriormente descrito representa el orden celestial y se ha denominado como Bāguà del Cielo anterior o Xiāntiān Bāguà 先天八卦. En esta compleja concepción bidimensional del espacio-tiempo, subyace un sistema de trazado geométrico cartesiano y polar, formado por números pares $(0,2,4,8)$ que implican la noción de un centro considerado vacío, o quizás no considerado. En este sentido, Lăozǐ comenta en el Dào Dé Jìng 道德经 que:

\section{道生一，一生二，二生三，三生万物。}

Dàoshēng yī, yīshēng èr, èr shēng sān, sān shēng wànwù.

El curso genera el uno, el uno genera el dos, el dos genera el tres, el tres genera todos los seres (Dào Dé Jìng, cap. 17. En Suarez 2009: 113).

Según la tradición china, del vacío absoluto surge espontáneamente la energía vital o $q i$ 气 (氣 en chino tradicional), que al ser considerado una unidad indivisible, constituye implícitamente una dualidad, dos elementos contrapuestos y complementarios, el yīn-yáng. Cuando se quiere situar la existencia del hombre en relación a la naturaleza surge la idea de centralidad y como consecuencia el concepto de uno, que ocupa un lugar central en equilibrio con las dualidades que componen el universo.

Por otro lado, según la cosmología China, el padre cielo fertiliza a la madre tierra con el qì y de la relación armónica entre ellos surge la multiplicidad y los seres humanos. Respecto a la relación entre el cielo y la tierra Lăož́ comenta:

\section{天下，万物生于有，有生于无。}

Tiānxià, wànwù shēng yú yǒu, yǒu shēng yú wú.

Bajo el cielo, todos los seres surgen del ser, el ser surge de la nada (Dào Dé Jìng, cap. 40. En Suarez 2009: 109).

El ser y la nada se consideran una dualidad que representa respectivamente al cielo y la tierra. De la unión entre ellos surge la armonía, que simbólicamente es irradiada por Shàngdì 上帝, el dios supremo del cielo, que gobierna la región central de la bóveda celeste. Del mismo modo, el emperador, su hijo en la tierra, recibe el mandato del cielo y desde el centro del país del centro o Zhōngguó 中国 (China) irradia su armonía generando el orden y la civilización. En este sentido, Guăn Zhòng 管仲 comenta en el Guănzí 管子 (período de Primaveras y Otoños): 


\section{天子中而处.}

Tiānž̀ zhōng ér chŭ.

El emperador (rey) siempre reside en el centro.

Por tanto surge el concepto de centro, ocupado por los gobernantes del cielo y de la tierra, que se representan mirando hacia el sur, con la izquierda situada al este y la derecha al oeste. Para reproducir esa correspondencia entre izquierda-este y derecha-oeste, el bāguà se orienta con el sur hacia arriba. Por el mismo motivo, los mapas chinos se han representado tradicionalmente también con esa disposición.

Como consecuencia, en la cosmología China se considera que el centro hace referencia a "el lugar hacia el que todo regresa, o todo converge, y, complementariamente, desde el que todo se difunde. El Centro (...) es el lugar del Hombre como arquetipo e instancia cósmica, santo soberano de la tradición china, o Santo taoísta. Es el espacio en que se entrecruzan el yīn y el yáng para producir el mundo, el lugar en que se sitúa el adepto en su meditación y el sacerdote en el ritual, desde y hacia el cual pueden comunicar con el Cielo igual que con la Tierra" (Robinet 2009: 95).

Como resultado de combinar el centro con la dualidad yinn-yáng surge el número 3. Del mismo modo, el 5 se derivó de añadir el centro a las cuatro orientaciones principales, constituyendo un número puro en la tradición china que representa el orden de la naturaleza. Así mismo, el 9 se ha formado combinando las ocho orientaciones que conforman el bāguà con el centro, $\mathrm{y}$ es considerado por tanto el número más relevante en la tierra. A partir de él, se han generado representaciones geométricas para reflejar el orden terrestre, que han sido utilizadas como base para generar patrones arquitectónicos y urbanos.

\subsection{Paralelismo entre el cielo y la tierra. El Xiāntiān Bāguà 先天八卦 y el Hòutiān Bāguà 后天八卦}

Combinando el esquema bidimensional contenido en el Bāguà que representa el orden celestial, con las ideas cosmológicas que relacionan el padre cielo con la madre tierra a través de un eje central ocupado por el gobernante del cielo y su hijo en la tierra, se obtiene un modelo tridimensional. En el Yijing se refleja el sentido de esta relación entre el orden celestial y el patrón terrestre. En concreto se comenta: 


\section{古者包爔氏之王天下也，仰則觀象於天，俯則觀法於地。}

Gǔ zhě Bāoxī shì zhī wáng tiānxià yě, yăng zé guān xiàng yú tiān, fǔ zé guān fă yú dì.

Antiguamente, cuando Bāoxī shì 包犧氏 llegó para gobernar todo bajo el cielo, miró hacia arriba y contempló las formas exhibidas en el cielo (las constelaciones), y miró hacia abajo para contemplar los procesos que tenían lugar sobre la tierra.

Se establece por tanto un patrón cosmológico, según el cual, existe un paralelismo entre el cielo y la tierra. En este sentido, se comenta también en el Yijing que:

\section{在天成象, 在地成形, 变化见矣。}

Zài tiān chéng xiàng, zài dì chéng xíng, biànhuà jiàn y̌̌.

En los cielos los fenómenos toman forma, sobre la tierra las formas se configuran. De este modo el cambio y la transformación se manifiestan.

Por otro lado, en la cosmología China se considera que el cielo está dividido en cuatro regiones que contienen cada una siete formaciones estelares. Estos cuadrantes están representados por animales simbólicos: el dragón turquesa (este), el pájaro bermellón (sur), el tigre blanco (oeste) y la tortuga negra en continua lucha con una serpiente (norte). Estas regiones, tienen su reflejo en la tierra, tanto en las estructuras naturales como en las realizadas por el hombre. Esta forma de pensamiento correlativo entre el cielo y la tierra, está asociada con la teoría del fēng shuǐ 风水 y es responsable del establecimiento de una estructura cósmica en el diseño arquitectónico.

El Bāguà del Cielo Anterior (Xiāntiān Bāguà 先天八卦), que representa el orden celestial, tiene por tanto una correspondencia en la tierra. Como consecuencia, se desarrolló el Bāguà del Cielo Posterior (Hòutiān Bāguà 后 天八卦), en el cual los trigramas están organizados con otra disposición para representar el orden terrestre. 


\subsection{Las representaciones cósmicas del Hétú 河图 y Luòshū 洛書 en relación al orden cósmico}

Los bāguà que representa el orden celestial y el terrestre, están a su vez asociados a tablas numéricas que correlacionan los fenómenos naturales. En concreto el Hétú 河图, presenta una geometría circular que simboliza los principios celestiales, y el Luòshū 洛書, dispone de una forma cuadrada que está relacionada con el orden terrestre. De este modo, responden a la concepción ancestral china de que el cielo es redondo y la tierra cuadrada. El origen del Luòshū y Hétú es todavía desconocido, pero tienen más de 3000 años de antigüedad, ya que son anteriores al Yijing. De hecho en el Libro de los Cambios se encuentran referencias al Luòshū.

En el Hétú, los números se disponen siguiendo los cuatro puntos cardinales y el centro, que como hemos comentado anteriormente, constituyen un esquema primario que representa el espacio y el tiempo. Los números pares hacen referencia al yin y se representan como puntos negros, mientras que los impares simbolizan el yáng y son dibujados en color blanco. En cada una de las cinco direcciones se sitúa un número par y otro impar, para conseguir de este modo el equilibrio entre el yīn y el yáng. De acuerdo a los principios celestiales, esta dualidad está en un continuo equilibrio dinámico. El yīn se mueve siguiendo el movimiento del sol, mientras que el yáng en sentido opuesto. Estos movimientos se bloquean en el eje nordeste-suroeste, que representa un estado inestable de yīn-yáng y por tanto desfavorable según el fēngshuǐ. Esta misma concepción se refleja en el Bāguà del Cielo Anterior, en el cual, los números asociados a trigramas opuestos suman siempre nueve, que constituye el número que mejor simboliza la tierra.

En base al número nueve se organiza como consecuencia el Luòshū, cosmograma que representa el orden terrestre y se ha convertido en una base filosófica para entender la estructura de la naturaleza. De hecho, se considera que ha inspirado la disposición de los 8 trigramas del $B$ āguà del Cielo Posterior, que son el resultado de reorganizar la disposición de los trigramas del $B$ āguà del Cielo Anterior. En este esquema matemático, los números impares o yáng, que ocupan un nivel jerárquico superior, se sitúan en los puntos cardinales, mientras que los números pares o yīn se disponen en las esquinas. Al igual que en el Hétú, en el centro se sitúa el número 5, que representa los cuatro puntos cardinales y la posición central. Sumando tres cifras alineadas en cualquier dirección (horizontal, vertical o diagonal), se obtiene el número 15.

El Luòshü, se puede representar como un cuadrado con 3 divisiones en cada lado, que conforman el Diagrama de las Nueve Cámaras o Jiǔgōng 九 宫. Cada uno de los 9 espacios resultantes está asociado a un número que corresponde con la organización del Luòshū. El Jiǔgōng y al Bāguà del Cielo 
Posterior están a su vez asociados con la Teoría de las Cinco Fases o wǔxíng 五 行. Sin embargo, son independientes en su origen. Los conceptos del yīn-yáng se utilizan para explicar los fenómenos cósmicos, mientras que la teoría de los Cinco Elementos representa la estructura física y conceptual del universo.

En la figura 2 se muestra el diagrama combinado del Xiāntiān Bāguà 先天 八卦 y Hòutiān Bāguà 后天八卦 (cielo anterior y posterior respectivamente) con sus diagramas asociados, utilizados para representar tanto los principios celestiales ideales como los principios terrestres prácticos. Reflejan por tanto el paralelismo entre el cielo y la tierra, que se traduce en una correlación numérica entre las diferentes orientaciones. Por otro lado, la relación entre el orden celestial y el terrestre con el Hétú y Luòshū respectivamente, implica que estos diagramas cosmológicos son aplicados en la arquitectura mediante el fēngshǔ para alcanzar la armonía entre el hombre y la naturaleza.

\section{Orden Celestial}

Xiāntiān bāguà 先天八卦

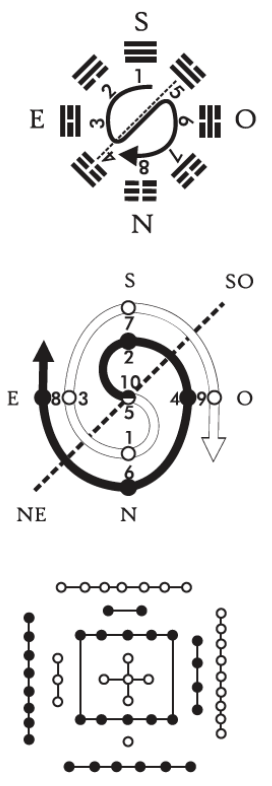

Hétú 河图
Orden Terrestre Hòutiān bāguà 后天八卦

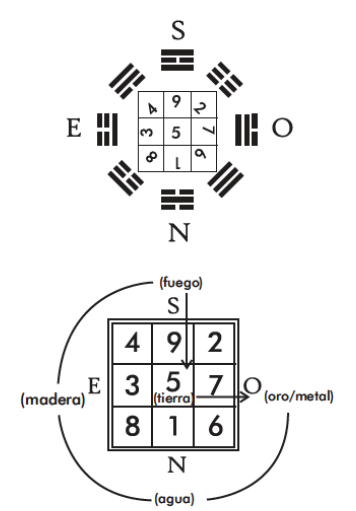

Jiǔgōng 九宫 - wǔxíng 五行

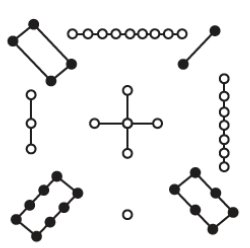

Luòshū 洛書

Figura 2. Relación entre el orden celestial y el orden terrestre. 


\section{Geometría cósmica. El sistema Bùjú 布局 y la generación de pa- trones arquitectónicos y urbanos}

\subsection{La estructura del Cosmos. Relación del todo con las partes}

El bāguà está compuesto por ocho trigramas que representan principios básicos de multiplicación y permutación basados en la dualidad del yīn y yáng. Las estructuras resultantes se disponen en el espacio y el tiempo siguiendo leyes cosmológicas que proporcionan armonía entre el cielo, la tierra y los seres humanos. Tal y como se ha comentado anteriormente, el proceso de multiplicación de dos a cuatro y ocho es el resultado de añadir a los módulos previos una dualidad yīn-yáng. En un nivel superior de organización, considerando los trigramas como módulo base de combinación y asociando los ochos trigramas de dos en dos, se generan los 64 hexagramas. Estos diagramas han sido analizados y utilizados en el Yijinng con fines adivinatorios. Debemos tener en cuenta que el término shù 数, utilizado en China para hacer referencia al concepto de número, significa también calcular o destino.

En esta forma concepción cosmológica, el todo, en este caso un hexagrama, contiene implícitamente las leyes de composición de los trigramas, que a su vez están constituidos por la dualidad yīn-yáng. Es decir, en cada nivel de composición, el todo contiene las partes, que paralelamente contienen potencialmente el todo. Como consecuencia, se establecen unas relaciones entre elementos o módulos que son derivados unos de otros y relacionados simultáneamente más allá de su jerarquía, para construir una estructura compleja que responde a las leyes cósmicas que proporcionan armonía con la naturaleza.

De forma análoga, en la ciencia se está utilizando recientemente un nuevo modo de inteligibilidad, ligado a la Teoría del Caos, que codifica la información según un sistema binario. Esta nueva forma de conocimiento, utilizada para hacer inteligible sistemas complejos, permite analizar las relaciones entre las partes y el todo mediante métodos de simulación por ordenador. El lenguaje utilizado se articula también en un sistema binario que permite codificar la información en 8,32 o 64 bits. 


\subsection{La dualidad Bùjú 布局 (macro-micro) como instrumento para la estandarización}

Esta forma de estructurar la realidad puede ser también representada mediante la dualidad bùjú 布局 (macro-micro), que sugiere la existencia de dos niveles de organización complementarios. El término Bù 布 representa el sistema de división desde lo macro a lo micro. A partir de la estructura global, que constituye el nivel de composición más elevado, se articulan hacia abajo limitaciones destinadas a que cada elemento ocupe el lugar, proporción y función adecuada. Por el contrario, el sistema jú 局 muestra los principios de crecimiento desde lo micro hasta lo macro. Se articula mediante el establecimiento de reglas de composición de las distintas partes o elementos para conseguir un todo armónico. Como resultado, cada parte contiene potencialmente el todo, que a su vez establece o regula las partes.

Esta forma de composición implica un gran número de multiplicaciones y permutaciones de módulos intercambiables en diferentes escalas o niveles jerárquicos, que responden a unas leyes o limitaciones preestablecidas. De este modo se ha resuelto uno de los problemas que ha obsesionado a la estandarización de la arquitectura. En concreto, cómo resolver la aparente contradicción de obtener gran cantidad de soluciones utilizando pocos módulos o variaciones. En este sentido, Frank Lloyd Wright señala que "el artista oriental percibe en todo el patrón, el artista occidental valores" (James, 1968). Por otro lado, en el Dao de la Arquitectura, libro en el que Amos Ih Tiao Chang cita profusamente a Wright, se comenta que:

Puesto que consta de un contenido intangible, una forma incompleta o fragmentaria preserva la posibilidad de adaptación flexible con las formas circundantes del mismo nivel de organización y, del mismo modo, se integra con ellas para llegar a ser una unidad completa de mayor escala sin destruir la continuidad de su medio. Para alcanzar la realidad de armonía y crecimiento, sólo es necesario mirar hacia arriba y ver en las estrellas, que no manifiestan su interrelación, qué bien parecen encajar esos puntos flotantes con cualquier forma construida por el hombre (Cabeza 2007: 56).

Como resultado, los patrones arquitectónicos desarrollados en China permiten una gran flexibilidad dentro de su rigidez compositiva, lo que facilita la versatilidad de soluciones a partir de pocos módulos o elementos. En este sentido, la tipología de casa patio puede ser usada para albergar a complejas y variadas estructuras familiares, templos religiosos, residencias oficiales, complejos palaciegos o incluso una gran ciudad. 


\subsection{El soporte dóugǒng 斗栱 y su relación con la dimensión del espacio}

En sintonía con esta forma de entender la arquitectura, considerada como un microcosmos compuesto por dualidades organizadas en diferentes niveles de composición que reproducen el macrocosmos, el nivel más básico de modulación se encuentra en el dǒugóng 斗栱, literalmente cabeza-brazo. Se trata de un tipo de soporte o capitel cuyo diseño se realiza en función del vano entre pilares, razón por la cual, está vinculado a la dimensión de las estancias.

El dǒngǒng está compuesto por elementos de madera de reducido tamaño que se combinan en estructuras más complejas, lo que facilita que las piezas más largas puedan ser reservadas para los pilares y vigas. Responde por tanto al principio de economía de medios que siempre ha caracterizado la arquitectura de un país superpoblado como China. En función de la distancia entre pilares, que influye en el peso que tienen que transmitir estos soportes, su disposición varía. Las distintas formas de composición del dǒugǒng aparecen perfectamente detalladas y estandarizadas en torno al 1100 d.C. en el Yíngzàofăshì.

\subsection{El concepto de espacio arquitectónico. Del jiān 间 en China al ma 間 en Japón}

En el segundo nivel de composición se sitúa el jiān 间, que consiste en un espacio rectangular rodeado por muros o definido por columnas que lo separan de espacios contiguos. El pictograma jiān 间 está actualmente compuesto por un sol y una puerta. Se puede entender etimológicamente por tanto que el espacio arquitectónico esté vinculado a la luz que entra a través de la puerta, que a su vez varía con el movimiento de los astros que rigen el paso del tiempo y el modo en que se van transformando las estancias. Podemos deducir en este sentido, que la experiencia o percepción humana del espacio ha estado tradicionalmente ligada a los ciclos naturales, que se manifiestan a través de espacios abiertos o patios.

En Japón se utiliza el mismo pictograma que en chino tradicional y se conoce como $m a$ 間, que significa también "entre" o espacio de transición. De hecho, la relación con el exterior se ha realizado tradicionalmente mediante una sucesión de espacios. Desde la fachada se proyecta al exterior un gran alero o noki 軒, tras el que se sitúa una galería o engawa 縁 que se cierra en invierno con paneles de shôji 所持, y posteriormente se dispone una sucesión de espacios interiores. Esta secuencia de elementos, que tiene un claro sentido climático, genera una gradación de luz desde el exterior hacia los oscuros espacios interiores, condicionando de este modo la forma de percibir y habitar el espacio. En las estancias se busca 
el vacío, caracterizado incluso por la ausencia de luz, que es llenado mediante la naturaleza representada por jardines exteriores, a los que se vinculan estrechamente las estancias. En este sentido, el paisaje exterior se ha utilizado frecuentemente como fondo paisajístico mediante la técnica denominada como shakkei 借景 o “fondos prestados”.

Conviene aclarar que en el budismo zen se considera que la contemplación de la naturaleza y el arte son una ayuda para alcanzar la iluminación, wù 悟 en chino y satori 悟り en japonés. En este sentido, zen es la pronunciación japonesa del budismo Chán 禅, tal y como se denomina en China, que a su vez deriva de la palabra sánscrita dhiana, que significa meditación.

El satori es considerado como un nivel de percepción superior que se alcanza mediante la meditación, eludiendo los condicionantes a través de la vacuidad, para alcanzar el conocimiento trascendente. Como consecuencia, para alcanzar la iluminación es de gran importancia el diseño de espacios neutros que respondan a los conceptos estéticos del wabi 侘び, sabi 寂 y shibui 渋い. Wabi hace referencia a la eliminación de todo lo superfluo, lo que pueda distraer en la búsqueda de la esencia. Sabi está relacionado con el paso del tiempo, que en Japón tiene un valor estético, y el shibui con la abstracción.

Este tipo de espacios neutros invitan a experimentar el misterio del vacío, a despegarse del mundo de lo fenomenológico o relativo, y a utilizar la naturaleza exterior como elemento catalizador para alcanzar el satori. El célebre maestro Matsuo Bashō 松尾芭蕉 describió este proceso magistralmente en el haiku 俳句:

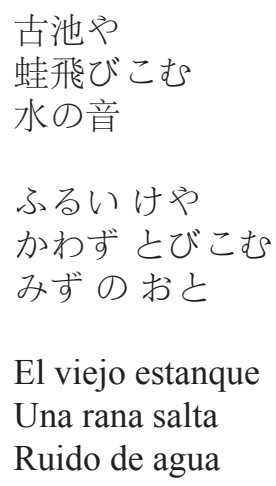

En este haiku, que refleja en sí mismo los principios zen, se establece una analogía entre el estanque y la conciencia humana. 
En definitiva, en Japón se ha entendido la geometría como un orden subyacente a la arquitectura que puede ser compatibilizado con la naturaleza. En este sentido, los espacios multifuncionales rectangulares que condiciona la rígida geometría del tatami, se han vinculado orgánicamente a los jardines.

Por el contrario, en China se dispone la edificación siguiendo leyes cósmicas que se traducen en una rígida geometría mediante la que se quiere establecer un orden simétrico respecto al eje norte sur, para conseguir de este modo el equilibrio entre el yīn y el yáng (oeste y este respectivamente). Como consecuencia, el hombre impone sus conceptos intelectuales de orden y armonía a la naturaleza.

\subsection{La composición de pabellones o tīng 厅. El sistema jiānjià 间架}

El concepto de Jiān, no sólo significa espacio, sino que también hace referencia a la luz de las vigas de carga. En el Yíngzàofăshì se considera el canto de una viga estandar de madera (cái 材) la unidad base de medida, a partir de la cual se deduce el resto de dimensiones de los edificios. Las vigas de atado situadas transversalmente se denominan jià 架. Los espacios o estancias se disponen de forma yuxtapuesta en sentido longitudinal y transversal para conformar edificios o tīng 厅. Este tipo de disposiciones, denominadas jiānjià 间架, facilita la estandarización y simplificación estructural de los edificios, lo que se traduce en rapidez de ejecución y economía de medios. Debido al alto grado de estandarización que presenta la estructura jiānjià, fue utilizada en el Yíngzàofăshì para categorizar diferentes tipos de estructuras en función del estatus social o político del propietario.

En este sentido, los pabellones o tīng disponen generalmente de 3 jiān, que representan la suma o conjunción del 1 o yáng y el 2 o yīn, y por tanto la armonía entre el yīn y el yáng. Cada espacio o jiān, recibe un nombre en función de su posición en el edificio. El espacio central se denomina iluminación o míng 明, mientras que las estancias contiguas a cada lado se conocen como $c i$ 次 o espacios secundarios. Salvo algunas excepciones, los pabellones presentan un número impar de jiān que no excede de once. De hecho, los pabellones de nueve jiān o excepcionalmente once, estaban reservados exclusivamente al palacio imperial.

Cuando se requiere un edificio de mayor superficie, se puede incrementar la dimensión de los jiān, aunque obviamente existe un límite debido a la resistencia estructural de los edificios, así como la posibilidad de mantener una estrecha relación con el exterior. Como consecuencia, el crecimiento del edificio se articula en base al incremento del número de pabellones o tīng. 


\subsection{La dualidad sólido-vacío como configuradora del fángzi 房子}

Los pabellones o tīng se disponen de forma armónica en torno a espacios abiertos o patios siguiendo leyes cósmicas para alcanzar la armonía con el orden natural. Los patrones arquitectónicos chinos reflejan como consecuencia una dualidad sólido-vacío, representada por el yuàn 院 o patio y el jiān o espacio construido. Como toda dualidad, constituye una unidad indivisible. En este sentido, se puede entender que:

Funcionalmente, el vacío sin sólido significaría retornar a una nebulosa salvaje. Visualmente, el sólido sin vacío implicaría la pérdida de la forma visible. Ninguna de ellas podría existir sin el complemento de la otra (Cabeza 2007: 56).

En esta dualidad, el vacío está asociado al yáng, que es superior jerárquicamente al yinn, representado por lo construido. Se formula por tanto un principio básico de composición según el cual el vacío o los espacios abiertos lideran una rígida combinación geométrica de edificios o pabellones, cuya razón de ser es su relación armónica con el exterior. Cuando un vacío o patio ha sido colmatado, la edificación se dispone en torno a otro vacío, convirtiéndose éste en el elemento articulador de la casa o fángzi 房子.

Este hecho supone una clara diferenciación con respecto a la arquitectura occidental, en la que en términos generales, existe una tendencia a complejizar la edificación aumentando las divisiones interiores. En China sin embargo, la complejidad radica en la organización de edificaciones simples para formar una estructura superior. Esta forma de entender la arquitectura explica que el foco de la composición sean los espacios abiertos o patios, en lugar del interior de los edificios.

Existen tres mecanismos que condicionan o limitan el crecimiento de las construcciones. El primero consiste en la extensión de la edificación a través del eje longitudinal norte-sur, conformando una serie alterna de llenos-vacíos, caracterizada por la dualidad yuàn-jiān. Cuando se ha agotado la posibilidad de prolongación a lo largo del eje norte-sur, debido frecuentemente a los límites constituidos por las calles orientadas en dirección este-oeste, el crecimiento de la edificación se realiza a lo largo de otro eje paralelo. El grupo de edificios y espacios vacíos en torno a este eje secundario alberga usos complementarios al del eje principal. La tercera posibilidad de crecimiento, consiste en prolongar un eje longitudinal o transversal de la edificación, generando espacios vacíos destinados generalmente a jardines privados.

En definitiva, los pabellones o tīng son muy simples y su tamaño está regulado y limitado por unas normas preestablecidas. El vacío o los espacios abiertos son los que articulan las edificaciones, que se sitúan en torno a ellos en armonía con la naturaleza para configurar los fángzi. Si la vivienda crece, no se 
complejiza o incrementa el tamaño de las edificaciones, sino que se aumenta el número de pabellones vinculados a espacios abiertos. En términos generales, se trata de una forma de proceder radicalmente diferente a la de occidente, donde para construir viviendas de mayor dimensión, se aumenta el tamaño de la planta y se subdivide con más particiones internas, lo cual limita su relación con el exterior.

\subsection{El esquema nonario de la ciudad o chéngshì 城市 y su relación con el sistema jǐngtián 井田}

El sistema de composición anteriormente descrito se aplica a viviendas pequeñas, grandes construcciones o incluso a una ciudad o chéngshi 城市. Para ello se tienen en cuenta obviamente las limitaciones o reglas propias del modelo de ciudad ideal. En China, la primera referencia escrita a cómo debe de ser el patrón urbano, aparece en torno al 475-221 a.C en el Kăogōngji 考工记 o Registro de los Artífices. En concreto se comenta que:

\section{匠人营国, 方九里, 旁三门, 国中九经九纬, 经涂九轨。}

Jiàngrén yíng guó, fāng jiǔlǐ, páng sān mén, guó zhōng jiǔ jīng jiǔ wěi, jīng tú jiǔ guĭ.

Los artificieros demarcaron la capital como un cuadrado con lados de 9 ľ, teniendo cada lado 3 puertas. Dentro de la capital había 9 avenidas meridionales y 9 latitudinales, teniendo cada una de las anteriores 9 vías para carros de ancho.

Se deduce, por tanto, que la estructura urbana ideal está configurada por un sistema nonario constituido por tres por tres lados. En este sentido, conviene señalar que, según la tradición china, el número 9 representa las ocho orientaciones principales junto con el centro y por tanto es el que mejor refleja el orden terrestre. Como consecuencia, se genera un sistema de composición jerarquizado, constituido por un nivel superior central rodeado por ocho módulos o elementos. Este concepto de centralidad subyace también en el diseño del eje principal norte-sur, ya que se dispone un número simétrico de calles paralelas a ambos lados, que representan el equilibrio entre el yīn y el yáng. En definitiva, el patrón urbano combina simultáneamente tres esquemas superpuestos. Un sistema cartesiano y otro polar derivado de un patrón reticular nonario, y un eje principal norte-sur que articula a los dos esquemas anteriores. 
Este sistema de composición fue utilizado posiblemente en las explotaciones agrarias antes de su aplicación al modelo de ciudad (Henderson 2011: 64). Debemos tener en cuenta, que la agricultura ha sido la base del sustento de la población, y que dada su importancia, ha estado especialmente regulada por los gobernantes. En concreto, se utilizaba un patrón nonario denominado jǐngtián 井田. El propio pictograma de pozo o jǐng 井, parece sugerir un esquema de tres por tres. En este sistema, la parcela central era común y se destinaba al pago de impuestos de los propietarios de las ocho parcelas ubicadas en torno a ella.

En definitiva, una ciudad o chéngshi se configura a partir de viviendas o fángzi, que a su vez están compuestos por tīng o pabellones formados por estancias (jiān) cuya dimensión condiciona la composición de los dǒugǒng. Este sistema de composición, denominado bùjú, relaciona las partes con el todo y el todo con las partes siguiendo leyes cósmicas para alcanzar la armonía entre el hombre y la naturaleza (figura 3).
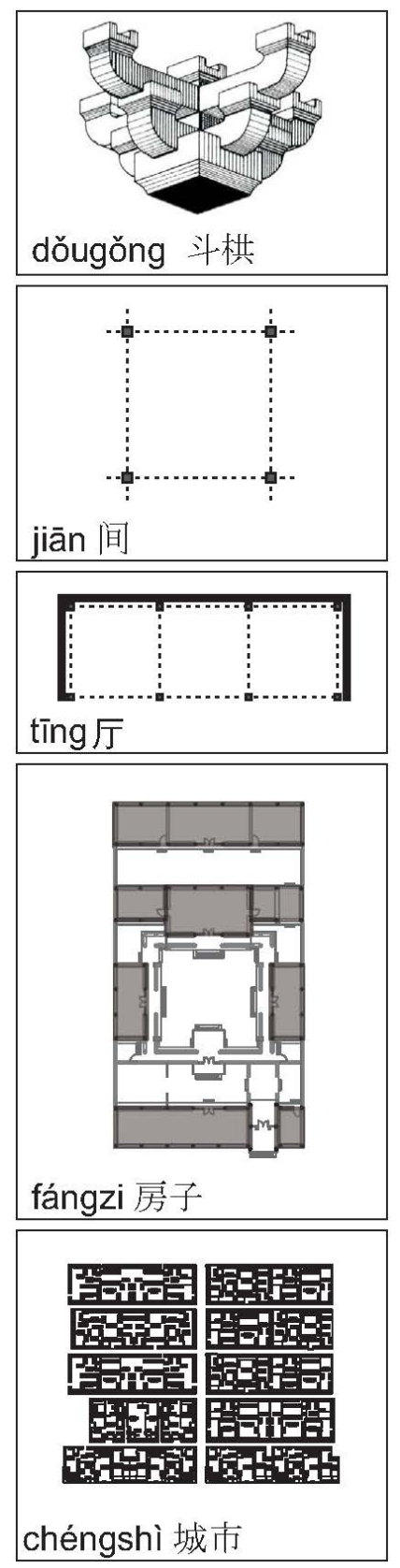

Figura 3. Sistema de composición bùjú 布局, macro-micro. 


\section{Jiā 家 o casa y familia. La vivienda como reflejo de la estructura social confuciana}

\subsection{Conceptos confucianos en relación a la vivienda y el orden cósmico}

La vivienda tradicional ha constituido un medio para reflejar conceptos confucianos en la sociedad. De hecho, casa o jiä 家 significa también familia, de modo que ambos conceptos han estado interrelacionados. Debemos de tener en cuenta, que dentro de la vivienda se generaba una micro-sociedad compuesta por una extensa familia estructurada mediante rígidas y complejas relaciones de jerarquía basadas en preceptos confucianos.

Un concepto clave en el paradigma social promulgado por Confucio es el rito o $l \check{l}$ 礼 (禮 en chino tradicional), que puede ser entendido como la gramática usada en las relaciones humanas. El lì regula los principios de orden o jerarquía y define el correcto comportamiento en relación a los roles sociales, entre los cuales ocupaban un papel muy importante las relaciones familiares. De hecho, de las cinco relaciones fundamentales o wǔlún 五论 establecidas por Confucio, tres de ellas se establecen en el ámbito familiar: padre-hijo, maridomujer, hermano mayor-hermano menor. Conviene mencionar en este sentido, que la base de la sociedad china no ha estado constituida tradicionalmente por el individuo, sino por la familia. De hecho, el bisílabo de país o guójia 国家, significa literalmente país-familia.

Por otro lado, se ha entendido tradicionalmente que el hombre debe de habitar la naturaleza en armonía con el cielo y la tierra, así como con la sociedad. Existe por tanto un paralelismo entre el orden cósmico y social que se refleja también en el bāguà. Este diagrama, no sólo establece una relación entre las energías de la naturaleza subyacentes en cada orientación, sino que además representa conceptos de orden ético o social confucianos. En concreto, las ocho direcciones se corresponden con ocho miembros de una familia formada por el padre, la madre, tres hijos y tres hijas. Como consecuencia, se establece una correspondencia entre la posición jerárquica de cada miembro de la familia y su orientación y ubicación dentro de la casa. La dirección que ocupa un nivel jerárquico más elevado es el sur, seguida del este, oeste y finalmente norte, la orientación más funesta según el fèngshuǐ. Esta jerarquía se corresponde con sus ventajas ambientales, tal y como se desprende de los resultados obtenidos en Pekín mediante el programa de análisis ambiental Ecotect (figura 4). 


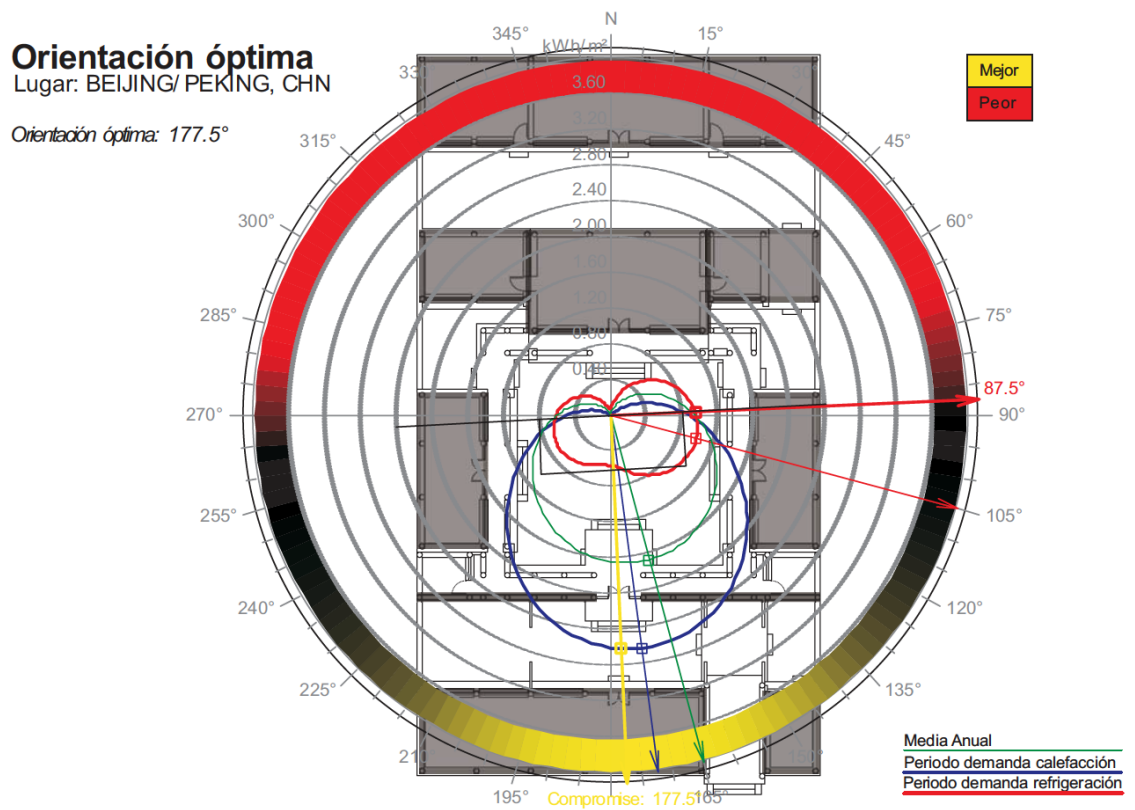

Figura 4. Orientación óptima en Pekín en función de la disponibilidad de radiación solar y las demandas de calefacción y refrigeración.

En este sentido, un dicho popular comenta que:

有钱不住东南房，冬不暖来夏不凉。

Yǒu qián bù zhù dōngnán fáng, dōng bù nuăn lái xià bù liáng.

Si tengo dinero no vivo en las estancias del este ni en las del sur, no son cálidas en invierno ni frescas en verano.

Las estancias situadas al este y al sur mencionadas en esta cita, corresponden con las orientadas al oeste y norte respectivamente, y por tanto con las que ocupan un menor nivel jerárquico dentro de la casa. Conviene aclarar que en determinadas circunstancias, los propietarios de las viviendas alquilaban estancias a otras familias, aunque siempre trataban de quedarse en las orientadas al sur. 


\subsection{Estructura espacial y jerarquía familiar}

El centro de la familia y por tanto de la casa, ha estado constituido por el pabellón de los ancestros. Al ser el edificio que ocupa la posición jerárquica más elevada, se ubica orientado al sur en el patio principal y con la viga de cumbrera dispuesta por tanto en dirección este-oeste. De hecho, el término utilizado para definir viga de cumbrera es dòng 栋, que se puede interpretar etimológicamente como un árbol o tronco orientado al este, lo cual es un indicativo de que ésta es la orientación ideal según la tradición china.

En la estancia central de este pabellón se sitúa el altar de los ancestros, que simboliza la unidad y continuidad de la familia, lo que tienen en común todos los miembros varones de la casa. En este sentido conviene aclarar, que la familia constituía una rígida sociedad patriarcal y patrilineal. Cuando las mujeres de la familia se casaban se iban a vivir con la familia del marido. Por tanto, en la vivienda sólo permanecían los miembros varones de la línea familiar.

Los miembros de la familia se ubicaban respecto al pabellón de los ancestros en función de su jerarquía. Mientras más cerca se situaba una estancia del pabellón de los ancestros, mayor era el estatus de la persona que la ocupaba, teniendo en cuenta que los pabellones orientados al este siempre tienen un nivel jerárquico superior a los que se disponen al oeste.

Los pabellones no eran nunca compartimentados en espacios intrincados, como generalmente ocurre en occidente. En el interior de los pabellones se disponían estancias simples yuxtapuestas con gran flexibilidad de uso. De hecho, para crear un determinado tipo de espacio sólo era necesario añadir los muebles requeridos. Por tanto, las estancias no se diferenciaban por su función específica, a excepción de la cocina y el pabellón principal.

Las paredes de la casa no eran portantes, debido a que se utilizaba una estructura de madera. Por tanto, existía una gran flexibilidad para poder abrir las estancias hacia los patios a través de grandes puertas pivotantes, huecos, celosías, etc. Por el contrario, la casa estaba muy cerrada hacia el exterior, donde se disponían pocas aberturas. Los pabellones adyacentes a las calles estaban además ocupados generalmente por sirvientes, almacenes, etc. Estos elementos actuaban como una barrera que protegía la intimidad de la familia de un mundo exterior caótico y desorganizado, hacia el que existía una fría indiferencia. En este sentido conviene recordar que en el wǔlún las relaciones sociales con extraños no están incluidas, y por tanto no están claramente definidas.

Por el contrario, en el interior de las casas se establecía una gran cooperación comunal y ayuda mutua entre los miembros de la familia. De hecho, la persona que ocupaba el primer lugar en la jerarquía, generalmente el varón más anciano, administraba la economía de todos los miembros de la familia. 


\subsection{Simbología cósmica y organización familiar. Interior-versus exterior}

La vivienda respondía simultáneamente tanto a ideas cosmológicas ancestrales, como al orden familiar establecido por conceptos confucianos. En China se ha establecido tradicionalmente un paralelismo entre el orden celestial y el modo en que el hombre debe habitar la tierra en armonía con el cosmos. Esta relación entre el macrocosmos y el microcosmos que se rige por los principios del fèngshul̆, literalmente viento-agua, ha condicionado tanto el emplazamiento y disposición ideal de una ciudad como el de una vivienda.

Según estos principios, el lugar ideal para ubicar una ciudad debe estar rodeado de montañas que simbolizan la protección de la madre tierra. Por otro lado, es conveniente la existencia al sur de agua en forma de lago o de un río sinuoso, así como de una montaña distante como fondo paisajístico, cuyo pico representa a los antepasados. De modo paralelo, los pabellones de la casa representan lo sólido o las montañas, simbolizadas por el yīn; mientras que el yáng (jerárquicamente superior) se identifica con al vacío ocupado por el patio. Como consecuencia, los pabellones situados al norte, este y oeste, representan las montañas protectoras, mientras que la entrada al patio principal simboliza el pico de la montaña delantera, asociada a los antepasados de la familia.

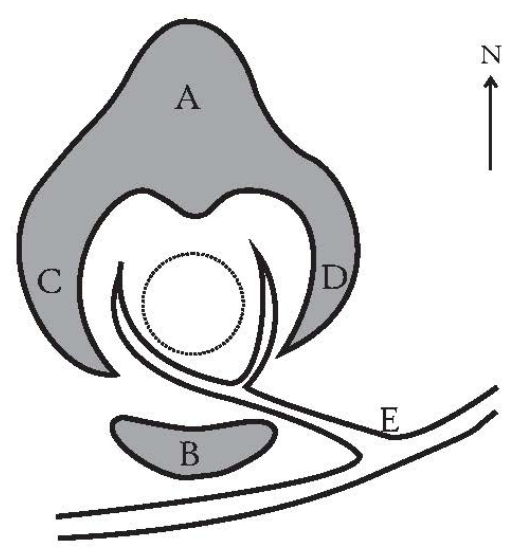
A: Tortuga Negra
B: Pájaro Bermellón
C: Tigre Blanco

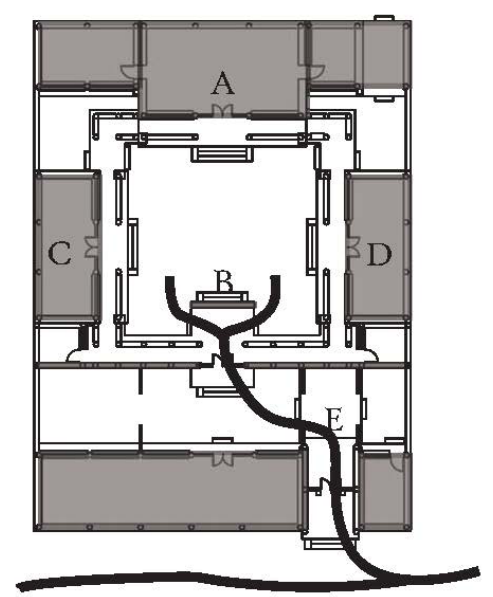

D: Dragón Turquesa

E: Entrada de Agua

Figura 5. Paralelismo entre el emplazamiento ideal para una ciudad según el fêngshǔ̌ 风水 y la configuración de una vivienda. 
La entrada de la vivienda se sitúa generalmente al sureste, que según el Bāguà del Cielo Posterior equivale al viento, a través del cual se desplaza el qì o energía vital. Tanto en la entrada a la vivienda como en el acceso a los patios se colocan muros pantalla o y̌ngbi 影壁 (literalmente muro sombra), que impide entrar directamente. Este recorrido de acceso sinuoso contribuye a privatizar la vida familiar a la vez que simboliza el curso de un río sinuoso, que según el fēngshuĭ deja pasar y acumula el qì positivo. Siguiendo este paralelismo entre el orden social y el orden cósmico, la vivienda se cierra al exterior tanto para salvaguardar la privacidad familiar, como para evitar la entrada del qì maligno, que según la tradición china, sólo se desplaza en línea recta, y por tanto, no puede penetrar a través del acceso sinuoso diseñado en las viviendas.

\subsection{El patio como símbolo de la naturaleza y elemento articulador de la vivienda}

En la vivienda tradicional, las estancias actúan como límites que permiten que el vacío o patio pueda ser identificado. Como consecuencia, el patio puede ser entendido como una habitación imaginaria que simboliza la naturaleza, en la que el cielo constituye el techo y la tierra el suelo. Debido a esta analogía, en los patios se plantaban frecuentemente árboles con un sentido simbólico. Los granados por ejemplo están asociados a la fecundidad o el ideal chino de tener una familia muy extensa. Por otro lado, los manzanos representan la armonía entre hermanos, algo que no es siempre fácil en familias muy numerosas y con unas rígidas relaciones jerárquicas. Sin embargo, nunca se colocan en un mismo patio dátiles zăo 疋 y peras $l i$ 梨, ya que su pronunciación (zăoli) es la misma que "pronto divorcio".

Como consecuencia, los patios o jardines son los elementos que articulan la vivienda y dan sentido a los espacios interiores a los que están estrechamente vinculados. El patio o vacío es por tanto concebido como el factor unificador que construye una estructura con la que el hombre se sitúa en la tierra en armonía con el cosmos. En este sentido Lăož̆ comenta:

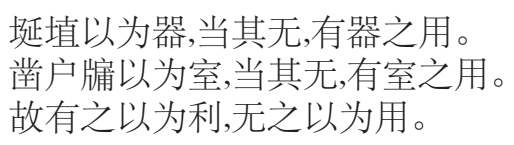

Shān zhí y̌̌wéi qì, dāng qí wú, yǒu qì zhī yòng.

Záo hùyǒu yı̌wéi shì, dāng qí wú, yǒu shì zhī yòng.

Gù yǒu zhī yı̌wéi lì, wú zhī yı̌wéi yòng. 
Se labra el barro para hacer vasijas, mas en su nada, radica la utilidad de la vasija.

Se horadan puertas y ventanas para hacer un aposento, mas en su nada radica la utilidad del aposento.

El ser es lo práctico, la nada es lo útil (Dào Dé Jing, cap. 11. En Suarez 2009: 51).

Se sugiere por tanto que el vacío es más importante que el ser, ya que permite que todas las cosas estén llenas para alcanzar su plenitud completa. A través del vacío ocupado por la naturaleza se produce el contacto directo del hombre con el movimiento de los astros, que determinan el paso del tiempo, las estaciones, el transcurso del día y la noche, en definitiva la experiencia del tiempo y del curso de la vida. En este sentido queremos destacar, que según la tradición china, la naturaleza está situada jerárquicamente por encima del hombre. Por otro lado, en relación al vacío Lăozľ comenta que:

\section{天下之至柔, 驰骋天下之至坚。 无有入无间,吾是以知无为之有益。}

Tiānxià zhī zhì róu, chíchěng tiānxià zhī zhì jiān.

Wú yǒu rù wújiān, wú shì y̌̌ zhī wúwéi zhī yǒuyì.

Lo más blando bajo el cielo, domina a lo más duro bajo el cielo.

Lo que no tiene ser penetra lo que no tiene intersticios, por eso conozco la ventaja de la no acción (Dào Dé Jing, cap. 18. En Suarez 2009: 115).

Podemos deducir como consecuencia, que el vacío ha estado ligado al concepto de no acción o wúwéi 无为 (無為 en chino tradicional). Wú significa nada o no ser, pero podría interpretarse al contrario, como no significar nada o ser. Como consecuencia, Wú puede ser entendido como la nada en el mundo fenomenológico, pero también como el todo, ya que del vacío surge el universo según la cosmología china y como consecuencia es lo primero, lo más importante.

En definitiva, los patrones arquitectónicos chinos reflejan una dualidad lleno-vacío que condiciona la forma en que el hombre se sitúa en armonía con el universo. El patio o vacío, que representa la naturaleza, constituye el elemento principal en torno al cual se disponen los edificios reflejando tanto el orden cósmico como el de la sociedad confuciana. 


\section{Conclusiones}

La arquitectura tradicional china ha estado ligada a conceptos cosmológicos en los que se establece un paralelismo entre el orden celestial y el patrón terrestre. En el marco de este pensamiento correlativo subyace la creencia de que el ser humano debe habitar la naturaleza en armonía con las leyes cósmicas que rigen el curso de los astros y la relación espacio-tiempo, siendo ésta la verdadera razón de ser de la existencia humana. Como consecuencia, se ha entendido tradicionalmente la arquitectura como un microcosmos que debe reproducir el macrocosmos.

En el Yijing, libro clásico que ha tenido una gran influencia en el pensamiento chino, se describe una concepción cosmológica según la cual, a partir de combinaciones en diferentes niveles de complejidad de la primera dualidad yīn-yáng se ha edificado el universo. Estas ideas aparecen representadas en el bāguà, esquema cósmico que define la posición que debe de ocupar el hombre como parte integrante de la naturaleza, para estar en armonía con el padre cielo y la madre tierra. Debido a esta relación entre el cielo y la tierra, se establece una equivalencia entre el bāguà que representa el orden celestial y su correspondencia en el orden terrestre, que a su vez están relacionados con otros esquemas geométricos y modelos matemáticos que han sido utilizados para explicar la estructura de la naturaleza. Entre ellos destacan el Hétú, Luòshū o Jiǔgōng.

De forma análoga al proceso descrito en el Yijing, los patrones arquitectónicos se han configurado mediante elementos simples que se relacionan según leyes cósmicas para configurar estructuras más complejas, que a su vez se multiplican y permutan entre sí siguiendo las mismas directrices para generar estructuras en un nivel superior y así sucesivamente. En sintonía con esta forma de entender la arquitectura en relación a leyes cósmicas, se considera como primer nivel de composición la dualidad dǒugǒng. Se trata de un soporte estructural cuya disposición está vinculada a la dimensión de los jiān o estancias rectangulares cuya combinación genera los pabellones o tīng. Estos se disponen en torno a patios generando los fángzi, cuya combinación en otro nivel superior de composición articula las ciudades o chéngshi.

Este sistema de composición obedece paralelamente al concepto Bùjú, que representa la dualidad macro-micro. El Bùjú sugiere la existencia de dos niveles de composición complementarios, siendo uno el reverso del otro. Bù hace referencia a la estructura de división desde lo macro a lo micro, mientras que $j u ́$ muestra los principios de crecimiento desde lo micro hasta lo macro. Como resultado de la combinación de ambos procedimientos, se consigue la relación armónica de las partes con el todo y viceversa. De este modo, la arquitectura tradicional china ha solucionado uno de los grandes objetivos 
de la estandarización en la arquitectura. En concreto, cómo obtener una gran cantidad de soluciones a partir de pocos módulos o elementos.

Por otro lado, la arquitectura ha sido tradicionalmente un reflejo del orden social y familiar, derivado de conceptos confucianos. La base de la sociedad china ha estado constituida por la familia, compuesta por un gran número de personas de varias generaciones que constituían una micro-sociedad. La organización espacial de la vivienda ha respondido a las rígidas y jerarquizadas normas que regían la estructura familiar. Podemos deducir por tanto, que la arquitectura china se fundamenta en el establecimiento de normas o patrones aparentemente simples, pero altamente elaborados a partir de concepciones cosmológicas, para conseguir armonía tanto con el orden natural como con el orden social.

Esta compleja y sofisticada forma de entender la arquitectura explica que el foco de la composición radique en el vacío, que representa a la naturaleza, al cual se vinculan las estancias de forma armónica siguiendo los cuatro puntos cardinales que representan la jerarquía y el orden familiar. La arquitectura china se sustenta por tanto más en la multiplicidad en términos de cantidad, que en la concentración en el interior o el volumen edificado.

En definitiva, los patrones arquitectónicos se han articulado en torno al vacío representado por la naturaleza, delimitándolo y apropiándose de él para reflejar el orden social, así como las leyes cósmicas representadas en el bāguà y recogidas en ideas de geomancia o fêngshuĭ. Se puede deducir por tanto que la arquitectura china sólo tiene sentido en relación a la naturaleza.

Para finalizar queremos destacar que China tiene una cultura milenaria que durante largos períodos de la historia de la humanidad ha tenido un desarrollo equiparable e incluso superior al de la cultura occidental. Desde las reformas económicas de finales de los 70, China ha experimentado un asombroso proceso de construcción y transformación socio-cultural sin parangón en la historia. En este sentido queremos destacar que en las últimas décadas se han construido en China más metros cuadrados que en ningún otro país y probablemente más que en el resto del mundo.

En este rápido proceso de transformación y apertura China está reconstruyendo su identidad, a la vez que el resto del mundo está mostrando un inusitado interés por su cultura milenaria, que como hemos puesto de manifiesto, presenta interesantes valores ambientales de los que se pueden extraer lecciones en occidente para avanzar hacia una arquitectura más sostenible. 


\section{Referencias bibliográficas:}

Cabeza, J.M. (2007). El Dao de la Arquitectura. Editorial Comares.

Henderson, J.B. (2011). The development and decline of Chinese cosmology. Windstone Press.

Rowe, P.G. and Seng, J. (2002). Architectural Encounters with Essence and Form in Modern China. The MIT Press, Cambridge, Massachusetts.

James, G. (1968). Frank Lloyd Wright's Imperial Hotel. New York: Dover Publications.

Knapp, R.G. (1999). China's Living Houses. University of Hawai Press.

Liu, Z. (2000). Typology and Structure of Chinese Architecture. China Architecture and Building Press, Beijing.

Robinet, I. (1999). Lao zi et le Tao, Bayard Jeunesse.

Sicheng, L. (2010). A Pictorical History of Chinese Architecture. SDX Publishing House.

Slote, W.H. (1998). Psychocultural dynamics within the Confucian family. State University of New York Press.

Steinhardt, N. et al. (2002). Chinese architecture. Yale University Press.

Suárez, A.H. (2009). Tao te King. Editorial Siruela, $5^{\text {a edición. }}$

Vilá, J. (2013). Yijing. El libro de los cambios. Memoria Mundi, Atlanta.

Zhang, W. (2004). The influence of Lao Tzu's "solid and void" on the Spatial Theories of Chinese Arquitectura. Journal of Tibet Nationalities Institute, vol. $25 \mathrm{n}^{\circ} .3$ : 62-64.

Zhu, J. (2004). Chinese spatial strategies: Imperial Beijing. Routledge Curzon. Taylor \& Francis Group. 
